\title{
A Test of Interventions to Increase Adherence to Hypertensive Medical Regimens
}

\author{
John P. Kirscht, Ph.D. \\ Jennifer L. Kirscht, M.P.H. \\ Irwin M. Rosenstock, Ph.D \\ University of Michigan
}

ABSTRACT: Low rates of adherence to hypertensive therapy limit patients' securing the tull benefits of treatment. While some tactors related to adherence have been identified, research on the effectiveness of interventions to increase adherence leve/s is sparse. The present study was designed to assess the impact of a series of different interventions on a group of some 400 patients, all under the care of private physicians in a small community. A tactorial design was employed to deliver four, sequential educational interventions, about four months apart, to randomly selected sub-groups. Interviews betore and atter each intervention provided information concerning self-reported adherence, health status, health beliets, and personal characteristics. Pertinent medical records and pharmacy data were also obtained. The tirst intervention-printed material-did not significantly attect adherence. The second and fourth interventions-nurse telephone calls and social support-each increased medication taking and the third intervention - self-monitoring - led to better weight control. There was no cumulative impact of the interventions and different aspects of regimens were not signiticantly related to one another.

Adherence to hypertensive regimens is widely recognized as a major problem. "Hypertension, considered a "silent" disease because it has few overt or specific symptoms, underscores the general and complex problem of patient compliance with medical recommendations. Considerable variation has been noted in the extent of compliance." Previous reviews also reflect the problem that few characteristics have been consistently demonstrated as related to compliance behavior. ${ }^{4}$

This research was supported by grant HL18401 from the National Heart, Lung and Blood Institute. Iohn Kirscht is a Protessor, University of Michigan School of Public Health, Department of Health Behavior and Health Education, 1420 Washington Heights, Ann Arbor, Michigan 48109. Jenniter Kirscht is a Research Associate, CRUSK, Institute for Social Research, University of Michigan. Irwin Rosenstock is a Protessor and Chairman, Department of Health Behavior and Health Education. University or Michigan. 
Given this absence of factors consistently associated with adherence, what suggestions have emerged for increasing patient adherence? We will briefly touch on only a few types of factors, including interpersonal relationships, knowledge, and situational arrangements and circumstances.

A number of studies, including some research on hypertension, point to the importance of patient-professional relationships." Higher levels of adherence are associated with patient perceptions of satisfactory interactions with providers.

The relationship of adherence to "education" or to knowledge of the medical condition is complex. Providing information to patients about their disease may not increase compliance. ${ }^{4}$ Sackett's research showed no significant increase in adherence with intensive, systematic education about the disease. ${ }^{6}$ Some knowledge of health conditions and of the reasons for the treatment, however, seem to be necessary for reasonable adherence. Covington and Pfeiffer found significant numbers of patients were poorly informed about drug therapy; McKenney concluded that intensive pharmacy services yielded better knowledge among hypertensive patients, which, in turn, mediated more effective adherence. ${ }^{8}$ Finnerty and Caldwell both concluded that better educational activities are needed for treatment of hypertension,' : and Wilbur and Barrow specifically cited the need for printed material in community programs. ${ }^{9}$ Haynes, Sackett, and others showed that personal attention and personal instruction about the regimen and taking blood pressure could significantly increase compliance. ${ }^{10}$

While little research has been done on situational determinants of compliance, especially those operating at the time compliance decisions are made, it seems reasonable to suppose that the behavior is influenced by various environmental contingencies. " One aspect of these is represented by the individual's social system. Since interpersonal influences loom large in everyday behavior, we would expect support by other family members to be important in adherence. Caplan's research found the social support system to affect dropout rate as well as an individual's view of the efficacy of treatment. ${ }^{12}$ As a negative factor, social isolation is related to noncompliance. A common pattern in illness behavior involves consultation with other family members. ${ }^{13}$ Social support, then, from the family would be expected to yield higher adherence to a regimen; Heinzelmann and Bagley found that the positive attitudes of spouses were associated with highrisk men staying in an experimental physical activity program. ${ }^{14}$

Related to situational determinants is the notion that behavior modification can be effected through arranging contingencies to 
evoke and reinforce desired behavior. However, these contingencies and rewards are not usually under the control of outside sources. Bandura makes the point that most behavioral repertoires are maintained by self-administered positive and negative reinforcers. ${ }^{15}$ Malahy's study of drug errors concluded that many patients lack a system for monitoring their medications. ${ }^{16}$ Any systematic reminder and recording arrangement should be of help. Knowledge of outcome, however, would be especially important. Wilbur and Barrow suggested selfmonitoring of blood pressure. ${ }^{9}$ Such monitoring would be expected to provide feedback on the results of adhering to therapy and thereby reinforce successful patterns of medication.

The present study was designed with two purposes in mind: (1) to investigate the dimensions of adherence as well as the relationship between adherence and an array of psychosocial factors; (2) to assess the impact of a series of interventions, varied in type, but all designed to have a positive effect on adherence. The present report focuses on the latter objective concerning the impact of interventions on adherence.

\section{STUDY DESIGN AND PROCEDURE}

Hypertensive patients of cooperating private practitioners (eight physicians in six practices) in Tecumseh. Michigan were enrolled in a three year study (1975-1978). At the beginning of the study, the investigators secured the cooperation of these physicians, all of whom were working in primary care practices. Two other physicians in the town were asked, but chose not to participate. Written invitations to serve in the study were sent by the physicians to all adult patients they were treating for high blood pressure. Of the approximately 700 who were contacted, 432 agreed to participate.

The definition of "high blood pressure" was whatever each physician so defined and no blood pressure cutoff level was utilized. The study focused on adherence, impact on blood pressure per se was not a purpose

Data were collected through a series of patient interviews conducted by trained interviewers, and supplemented by information obtained from medical and pharmacy records, for which written patient consent had been obtained. The interview data included measures of personal characteristics, knowledge about hypertension and its treatment, health beliefs and practices, and self-reported compliance behavior.

Medical and pharmacy record information was collected during a baseline period of about nine months. On the study consent forms, participants listed the pharmacies from which they obtained prescription medicines. Most reported purchasing their drugs at one of a few local pharmacies, which allowed collection of reliable information on prescription filling behaviors. After an initial interview, the participants were randomly assigned to each of four sequential intervention experiments. Interviews were then conducted after each intervention, for a total of five rounds of interviewing. An average of four months elapsed between each of the interventions. 
Four educational and behavioral intervention strategies were tested in a factorial design. The interventions were:

1. Printed messages. Participants were randomly assigned to one of three conditions: a threatening message group, a positive message group, or a control group. Two informational tabloids were developed and delivered to the experimental participants. Each contained material on hypertension, its effects, control measures, and instructions on following a regimen. One version emphasized the severity of high blood pressure and its potential consequences while the other emphasized positive health aspects of treatment.

2. Nurse reminder and reinforcement. Patients were randomly assigned to treatment or control. A study nurse called each participant in the treatment group, discussing the regimen and reinforcing any positive steps the patient was already taking. The call was preceded by a letter outlining the purpose and topics of the contact. Control subjects received no intervention.

3 Seli-monitoring. There was random assignment to three groups, including two experimental conditions and a control group. In the first, patients kept a two week set of daily charts on behaviors involved in the regimen (e.g., charting pills each day) using specially developed printed forms. In the second, patients were trained and then requested to take and record blood pressure readings daily for two weeks utilizing a cuff provided. Each participant in the intervention group was visited to initiate the monitoring process. Study interviewers were trained to carry out these initial visits. In the blood pressure cuff group, a nurse checked on proper use of the cuff during the intervention period. Control group participants received no intervention.

4. Social support. In this intervention, patients were randomly assigned to control or treatment. In the treatment condition, following a letter inviting participation, nurses from the study made appointments by phone to see each patient and a person selected by the patient who was to help the patient with problems encountered in following the regimen. The nurse visited the respondent and support person, discussed the purpose of the intervention, obtained a commitment that the pair would work on particular aspects of the regimen and staged a role played example. Two weeks later, the nurse called both the patient and the support person to give reinforcement and to answer questions. Control subjects received a letter thanking them for continued participation in the study, but were not otherwise contacted.

The four interventions, as indicated, were used in a sequential factorial design. Each group (e.g., those assigned to receive the positive communication in the first intervention) was subsequently randomized again for the next intervention. For the whole study, 36 groups were ultimately formed $(3 \times 2 \times 3 \times 2)$. Thus, some patients received all four interventions, some three, two, one or none. The sequence of the interventions was the same for all patients, whether or not they received one or more of the experimental treatments. The rationale for presenting interventions in this sequence was that it represented a logical progression from impersonal to personal. Also, this progression corresponded to an increase in patient involvement in the intervention itself, which was higher in the last two interventions (Self-Monitoring and Social Support) than in the first two interventions (Written Messages and Nurse Reminder). 
Originally, 432 patients agreed to participate in the study, and were interviewed at baseline. Of these, 417 were identified as clearly under treatment for hypertension. (Although all patients were identified by the medical practices as being treated for hypertension, some were found not to be under treatment for the condition). In the course of the study, over the period of $1 \frac{1}{2}$ years, some 54 patients were lost due to death, moves, or changing to physicians who were not participating in the study; 12 more declined to continue at some point. Thus, 351 people were in the study through the four interventions. The numbers participating varied with each intervention and interview. A major loss in the final intervention occurred because of the considerable time involved in carrying out the procedures: a number of those assigned to the intervention simply could not be reached within the period allotted or the participation of the support person could not be scheduled during the planned period of the intervention. Comparisons of characteristics of patients lost to the study with those who remained did not reveal any significant differences. In particular, patients who were assigned to a given group, but did not participate were compared with participants on prior adherence scores. Only at the last intervention was there a near significant difference. The possibility of selection bias was alleviated by use of covariance analysis.

Respondents were nearly all white, most $(60 \%)$ had attained a high school education, and most of those currently employed worked in blue collar jobs. The median family income was $\$ 12,000$ per year, with $42 \%$ earning less than $\$ 10,000$ per year. Most $(78 \%)$ were over fifty years of age.

Over $87 \%$ of the 417 hypertensive patients were on prescribed medication regimens, $47 \%$ had dietary restrictions (primarily sodium) and $41 \%$ had weight loss prescribed about one in five was prescribed all three kinds of regimens simultaneously. Most of the patients $(57 \%)$ had been diagnosed as hypertensive more than five years previously, and only $11 \%$ had been diagnosed within the prior year.

\section{Measures}

Information from three sources was used in this study. Patient interviews were conducted initially and after each intervention. The interviews, done in the patient's home, included questions concerning adherence to the medical regimen for hypertension, beliefs about the condition and a variety of other health matters, current living situation, social characteristics, and health status. Additionally, information about the regimen was obtained from the treating physician's records, along with information on number of visits and blood pressure. Pharmacy records were also consulted, providing data on amounts of prescribed medications obtained by the patient during each time period.

The major aspects of the regimens included medications, dietary restrictions, and control of weight. While other elements were included in the advice received by some patients, such as not smoking, stress reduction, or control of alcohol consumption, very few actually had a written regimen covering items other than medication, diet, or weight control. Hence, the results on interventions are restricted to those three classes of behavior.

Dietary adherence and weight control were assessed entirely through selfreport, while medication adherence was measured both through self-report and records. In the interview, the patient was asked "Have you been able to follow your doctor's advice? For example, how many pills did you take yesterday? The last time you didn't take the medicine, what was the reason? How of ten does this 
happen?" Similar items were used for other aspects of the regimen. From the answers, each patient was coded on an ordinal scale of adherence from low to high for each element in that patient's regimen. From the pharmacy records, an estimate was made of the number of daily doses of each prescribed medication. This quantity was divided by the number of days for which the prescription was in force. Averaged over the set of hypertensive medications, this ratio assessed the proportion of pills available to the patient.

\section{RESULTS AND DISCUSSION}

At baseline, some $42 \%$ of the group on hypertension medicines reported complete adherence in taking the prescribed pills. Pharmacy records, available for 345 of the patients for the baseline period, yielded a mean percentage of pills available of $63 \%(100 \%$ would mean all pills available for the periods prescribed). In terms of dietary restrictions, among the 198 participants whose regimen included this element, $26 \%$ reported virtually complete fidelity to the diet. Of the 182 patients on weight control, $12 \%$ reported they successfully achieved desired weight reduction; over $24 \%$, however, had been unable to lose any weight. According to these data, then, the study participants were not exceptionally high on adherence measures, and relatively lower levels of adherence were noted for diet and weight control. For all aspects of the regimens, there was, on the average, room for improvement.

Sociodemographic characteristics were, for the most part, unrelated to adherence. Adherence itself, however, proved to be a multidimensional concept; adherence to any element of the regimen did not predict level of adherence to any other element.

\section{Etfects of the Interventions}

It should be noted that the analyses of the effects of each intervention tested the differences between experimental and control groups on each measure of adherence after partialling out pre-experimental differences on the measure. This controlled for initial differences on the dependent variables.

Printed tabloids. The initial intervention involved provision of written information about hypertension in two different forms to randomly selected subgroups of the population. Analyses of variance of the major measures of adherence compared the two groups receiving the messages with the control group, since the two message groups did not differ from one another on any aspect of adherence. Table 1 presents the summary adherence scores for the groups. Taking baseline scores into account, medication scores (both pharmacy and self-report) an weight control scores were not 


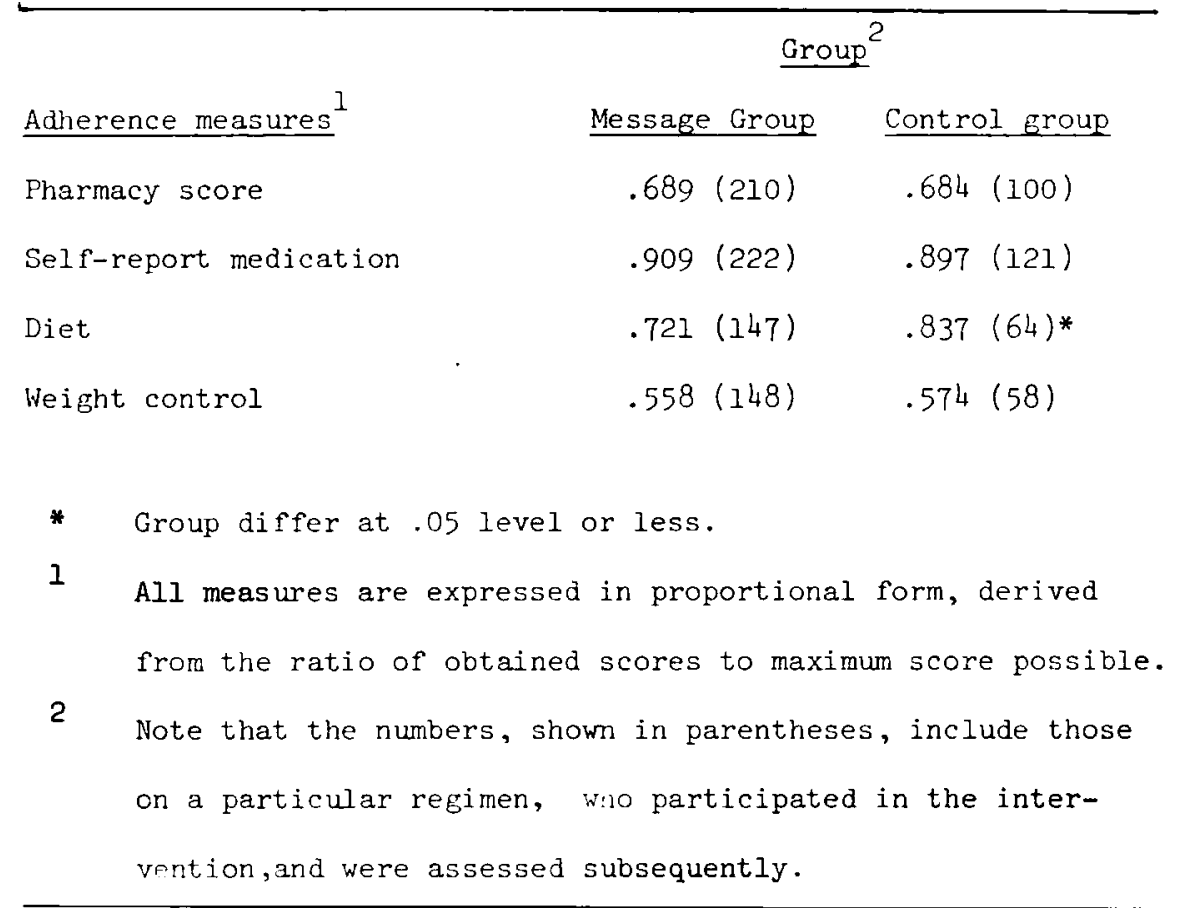

affected by the intervention. Self-reported diet scores, however, did differ significantly, although, unexpectedly, the control subjects had higher scores. The only positive effect of the messages was a significant increase in information about hypertension, as measured by a set of items in the interview, for the persons receiving the printed materials. The information scores were not associated with adherence.

Nurse-initiated telephone discussion. Analyses of the effects of the nurse phone contact, the second intervention, involved comparison of adherence scores of the randomly assigned half of the sample receiving the nurse call with the other half serving as controls. (Table 2). Given the previous intervention, however, it was also possible to divide the total group into the 6 subgroups of the crossed design $(3 \times 2)$ in order to find out if the initial intervention had any delayed effect or interacted with the nurse intervention.

The nurse telephone call resulted in a significant increase in medication adherence. With baseline scores taken into account, there was a significantly higher pharmacy score for the group receiving the phone discussion with the nurse. Moreover, selfreported medication scores were also significantly higher for the 
TABLE 2. Nurse Telephone Contact (Intervention 2) and Measures of Adherence in Experimental and Control Groups.

Group

Adherence measures

Pharmacy score

Self-report medication

Diet

Weight control
Nurse contact

$.749(129)$

$.957(148)$

$.720(82)$

$.447 \quad(90)$
Control group

$.690(141) *$

$.908(168) *$

$.754(100)$

$.465(108)$

intervention group. No effects were found, however, for the diet and weight control measures. The effects of the nurse phone call were maintained, when tested by factorial analyses of variance, using the full $2 \times 3$ design; there was no delayed effect on the first intervention nor did it interact with the nurse phone call.

Self-monitoring. The third intervention, involving self-monitoring either by recording regimen-related activities (one-third of the subjects) or taking and recording one's own blood pressure (another one-third of the participants), showed significant effects on the weight control measure, but not on the other measures (Table 3). There was a significant improvement in weight control for the intervention group, with most of the effect occurring among the participants who used the blood pressure cuff.

No interactions were found between this and any prior interventions on any dependent variable, when tested by factorial analyses of variance.

Social support. The final intervention entailed a social support maneuver with about half the participants, with the remainder serving as controls. This social support intervention significantly increased medication adherence (Table 4). Pharmacy records and self-reported medication scores were significantly higher for the group receiving the intervention, with baseline levels of these variables taken into account. There was also a trend for the intervention to have a positive effect on weight control. Dietary adherence scores, however, were not affected.

Analysis of the effects of the combined interventions did not yield significant results. This finding resulted from analysis of 
TABLE 3. Self-Monitoring (Intervention 3) and Measures of Adherence in Experimental and Control Groups

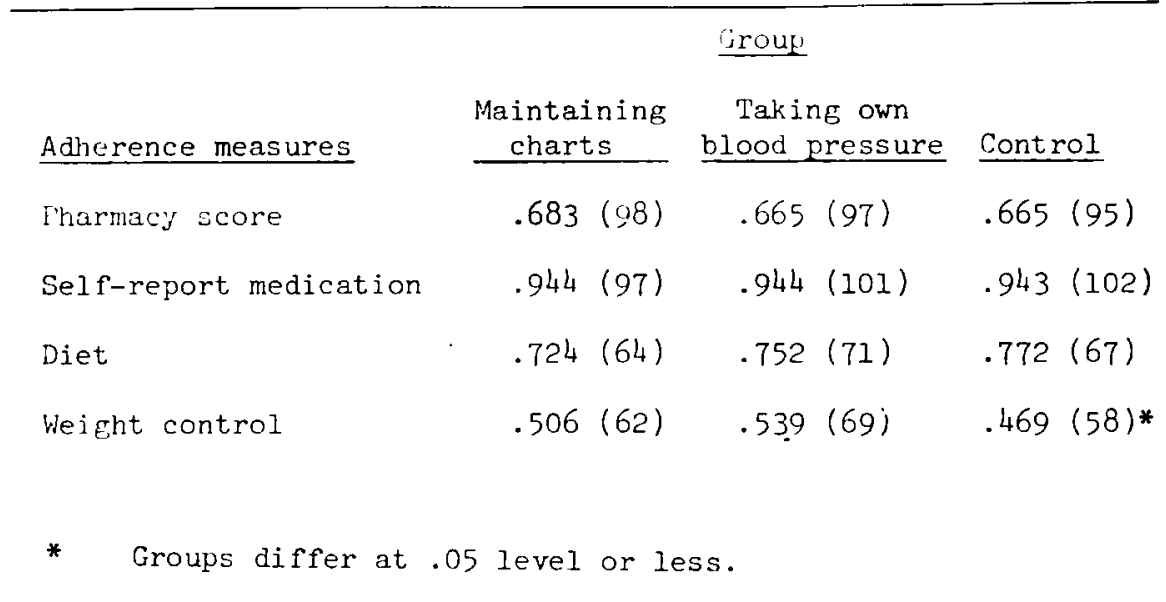

TABLE 4. Social Support (Intervention 4) and Measures of Adherence in Experimental and Control Croups

Groue

Adherence measuras

Pharmacy score

Self-report medication

Diet

Weight control
Social support Control group

$.654(96)$

$.545(139)^{*}$

$.978(97)$

$.934(131)^{*}$

$.686(70)$

$.784(88)$

$.482(70)$

$.408(96)$

* Groups differ at .05 level or less.

variance on groups formed from the combination of interventions 2,3 , and 4 ( 12 groups). Intervention 1 was omitted because of the lack of any apparent effect of this intervention and because of small numbers of cases in some groups where all four interventions are viewed simultaneously. The lack of any major results beyond simple effects of the particular interventions indicated that there was no cumulative impact of the sequence of interventions. Hence, participants who received both the social support and the self-monitoring were no more likely to exhibit higher medication scores than those who had social support but not self-monitoring.

The present study highlights the fact that adherence is a multidimensional concept and as such is a complex issue to 
examine. This is exemplified by the lack of correlation among the three components studied (medications, dietary restrictions, and weight control). The effects of the interventions also reflect this differentiation of adherence behaviors. Each intervention had some effects but they were limited and specific. No intervention increased adherence generally. The initial intervention, involving the provision of information through printed messages, had no discernible effects on adherence. That result is consistent with findings from other studies ${ }^{3}$ : a one-time presentation of material in an impersonal form will not affect adherence. Each of the other interventions was personalized to some degree and the most effective involved personal contact by a health professional.

Potential theoretical differences among the interventions were apparently not of much consequence. Although it is not immediately obvious why the nurse phone call and the social support interventions affected medication compliance rather than other aspects of the regimens, one hypothesis is that it is an easier behavior to change. That self-monitoring affected weight control is perhaps more understandable in that the form of the intervention may have called attention to self-regulated behaviors. It is possible that the use of the blood pressure cuff seemed to be more effective than charting of behaviors because it gave a direct link to the "silent" disease and it was more in line with what took place in the doctor's office and seemed more "official." The amount of effort put into the various interventions, and the amount of demand made on the patients did not seem to be reflected in the effects. In fact, from the point of view of outcomes, the phone discussion by the nurse was not only relatively simple and inexpensive to conduct, but also was not costly in time or effort for the patient. One possible mechanism through which 'medication adherence improved is that of contact between patient and physician. From records, we found that the nurse phone call and social support treatments were associated with more visits for hypertension related purposes.

An important limit on the effects of the interventions was the fact that they faded away and did not yield any apparent cumulative impact. Again, the temporal aspect of the effects is consistent with other findings in the literature that influences on adherence tend to be lost fairly rapidly. ${ }^{17}$ Since adherence to chronic disease regimens usually involves long-term, sometimes lifelong behaviors, one-time interventions should not be expected to produce long-term effects. ${ }^{18}$ Reinforcement or institutionalization of change may well be necessary for continued effects.

There are a number of limitations in the present study. 
Internally, the research involved repeated data collection from the group of patients - the interviews themselves constituted potential interventions to an unknown degree. Data collection periods were selected more or less arbitrarily since virtually no information is available as to how long one might expect an effect of an intervention to last. Further, the interventions all came from the outside. They were not built into or identified with the medical care system serving the patients. None of the usual care-givers was involved in the attempts at influencing patient behavior.

The results of the study add to information about ways of increasing patient adherence, both in pointing to the need for more differentiated intervention concepts and in indicating that some modes of changing patient behavior may be more effective than others. Where treatment involves a series of different behaviors, such as medications, dietary modification, and exercise, more than one intervention strategy may be required to increase levels of adherence. At the same time, the outcomes call attention to the need for considering the longer term effects of efforts at change.

\section{SUMMARY AND CONCLUSIONS}

The results of this study increase our knowledge about ways of increasing patient adherence. We found that one-time printed informational message did not have a positive effect on adherence while a single nurse-telephone contact increased medication adherence, a self-monitoring intervention increased weight control, and a social support intervention increased medication adherence. Several points of theoretical and practical significance are implied by these findings. For one thing, in the present case, at least, personal approaches to patients had more effect than the impersonal approach. Secondly, the three successful interventions influenced different components of adherence. Third, even the successful interventions did not have long-lasting effects. Because the nurse-telephone call was the least expensive in time and money of all the successful interventions, further investigation should be made of nurses potential in increasing adherence to particular elements of medical regimens. These results are all consistent with the conclusion that adherence is a multidimensional concept, requiring different interventions for modifying different components of adherence. Effective strategies for increasing compliance may well have to incorporate plans for repeated administrations of the intervention, in contrast with one-time efforts to modify behavior. 


\section{REFERENCES}

1 Caldwell J. Cobb S. Dowling $M$, Jongh D The dropout problem in antihypertensive therapy / Chronic Dis 22:579-592, 1970.

2 Finnerty F Ir, Matte E, Finnerty F III. Hypertension on the Inner city I Analysis of clinic dropouts Circulation 47:73-75, 1973

3 Haynes R. Taylor D. Sackett D (Eds): Compliance in Health Care. Baltmore, Johns Hopkıns University Press, 1979

4 Kirscht J, Rosentock 1. Patients problems in following recommendations of heath experts. In C Stone. F Cohen \& N Alder (Eds). Health Psychology. San Francisco, JosseyBass, 1979

5. Hulka B: Patient-clinician interactions and compliance. In R Haynes, D Taylor, \& D Sackett (Eds). Compliance in Health Care. Baltimore, Johns Hopkins University Press. 1979.

6 Sackett $D$ et al Randomized clinical trial of strategies for improving medication compliance in primary hypertension Lancet 1:1205-1207, 1975.

7 Covington T. Pfieffer F. The pharmacist-acquired medication history Amer / Hosp Pharm 29:691-695, 1972

8 Mckenney et al: The effect of clinical pharmacy services on patients with essential hypertension. Circulation 48:1104-1111, 1973

9. Wilbur I, Barrow I; Hypertension: A community problem Amer / Med 52:653-663, 1972.

10. Haynes $R$ et al: Improvement of medication compliance in uncontrolled hypertension. Lancet 1:1265-1268, 1976

11 Dunbar I. Marshall C. Hovell M: Behavioral strategies for improving compliance In R Haynes, D Taylor, \& D Sackett, (Eds) Compliance in Health Care. Baltimore, lohns Hopkins University Press, 1979

12 Caplan R et al: Adhering to Medical Regimens. Ann Arbor, Research Center for Group Dynamics, Institute for Social Research, University of Michigan, 1976.

13 Twaddle A: Health decisions and sick role variations: An exploration / Health \& Social Behav 10:105-114, 1969

14 Heinzelmann F. Bagley R: Response to physical activity programs and their effects on health behavior Pub Health Rep 85:905-911, 1970.

15 Bandura A: Principles of Behavior Moditication. Englewood Cliffs, NI. Prentice-Hall, 1969

16 Malahy $B$ : The effect of instruction and labelling on the number of medication errors made by patients at home Amer / Hosp Pharm 23:283-292, 1966

17. Kasl S: A social psychological perspective on successful community control of high blood pressure / Behav Med 1:347-381, 1978.

18 Green L: Educational strategies to improve compliance with therapeutic and preventive regimens: The recent evidence In R Haynes, D Taylor \& D Sackett (Eds). Compliance in Health Care. Baltimore, Johns Hopkins University Press, 1979. 